\title{
Novel method to synthesis nickel oxide nanoparticles for antibacterial activity
}

\author{
A M Rheima', A A Anber², A A Shakir ${ }^{3}$, A S Hameed ${ }^{4}$, and Sh A Hameed ${ }^{1}$ \\ 1. Department of Chemistry, College of Science, University of Wasit, Wasit, Iraq \\ 2. Department of Student Affairs and Registration, Al-Karkh University of Science, Baghdad, Iraq \\ 3. Department of Chemistry, College of Biotechnology, Al-Qasim green University, Iraq \\ 4. Department of Physics, College of Science, University of Kerbala, Karbala, Iraq \\ E-mail: Ahmedkrm88@gmail.com
}

(Received 24 August 2019 ; in final form 22 January 2020)

\begin{abstract}
In this work, nickel oxide nanoparticles (NiO NPs) have been synthesized via photo irradiation as a novel method. It is a simple and cost effective method. The average particle size and morphology were examined by AFM, TEM, SEM and the crystallinity was estimated by XRD analysis and the strength of magnetic field of samples were measured by using gaussmeter device. XRD studies confirm the nickel oxide nanoparticles have a high degree of crystallinity nature. Their particle size of nickel oxide was found about $12 \mathrm{~nm}$. The values inhibition zone indicates that nanoparticles effect on different bacteria. The outcome considered a new synthesis of $\mathrm{NiO}$ nanoparticles to promise antimicrobial agents against bacteria.
\end{abstract}

Keywords: nickel oxide, photo irradiation, antimicrobial

\section{Introduction}

Nanotechnology represents one of the most active research areas in modern techniques. The applications of nanoparticles is increasing in recent years due to unique their chemical, physical and biological properties [1-4]. Nowadays the synthesis of metal nanoparticles have received increasing attention due to their extremely application such as ,magnetic ,electrical, thermal sensor devices , chemical and biological properties especially catalytic properties which is not achieve from those in bulk materials [5-7]. Nickel oxide is one of the nanoparticles of intermediate metal oxide. It is also a material with various applications that is semiconductive [8]. It has a high area and unique characteristics therefore the scientists are very concerned about these nanoparticles due to the nanoparticles of nickel oxide contain low toxicity and biocompatibility [9].

Nickel oxide nanoparticles an important class of martials because of their important both in technology and science, it's well known that specific properties of inorganic materials, nickel oxide has different stable oxidation state, they have corrosion resistant, high temperature resistant, crystal displays, liquid, catalysts, and so on. So far many different techniques have been developed to synthesis these metal in nano scale such as sol gel, micro emulsion, chemical methods, the reproducible and controlled synthesis of stable and defined metal nanoparticles with small size is so important for arrange of applications especially in medicine, the small size of nanoparticles and large surface area to volume ratio put nanoparticle in position for wide application essentially in biomedicine [10-13]. The antibacterial activity of silver, gold and plant extract copper nanoparticles has been determined due to high photosensitivity, environmentally friendly, non-toxic and low cost. Metal oxide NPs were used for photocatalytic environmental remediation such as wastewater treatment, this catalyst, however, involves photon absorption to produce pairs of electrons (e-) and holes $(\mathrm{h}+)$ in aqueous solution [14-17]. Nickel oxide nanoparticles have high strong bacterial activity [18], against gram negative and gram positive bacteria [1922].

Therefore, in the present work, we have synthesized and investigated nickel oxide nanoparticles (NiO NPs) via photo irradiation as a novel methods, were examined by AFM, TEM, SEM and the crystallinity was estimated by XRD analysis, and antibacterial properties of $(\mathrm{NiO}$ NPs) thin films. 


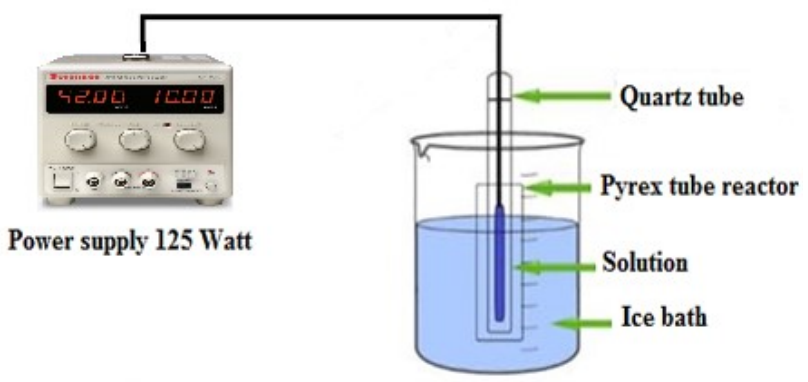

Figure 1. Photo irradiation method (irradiation cell).
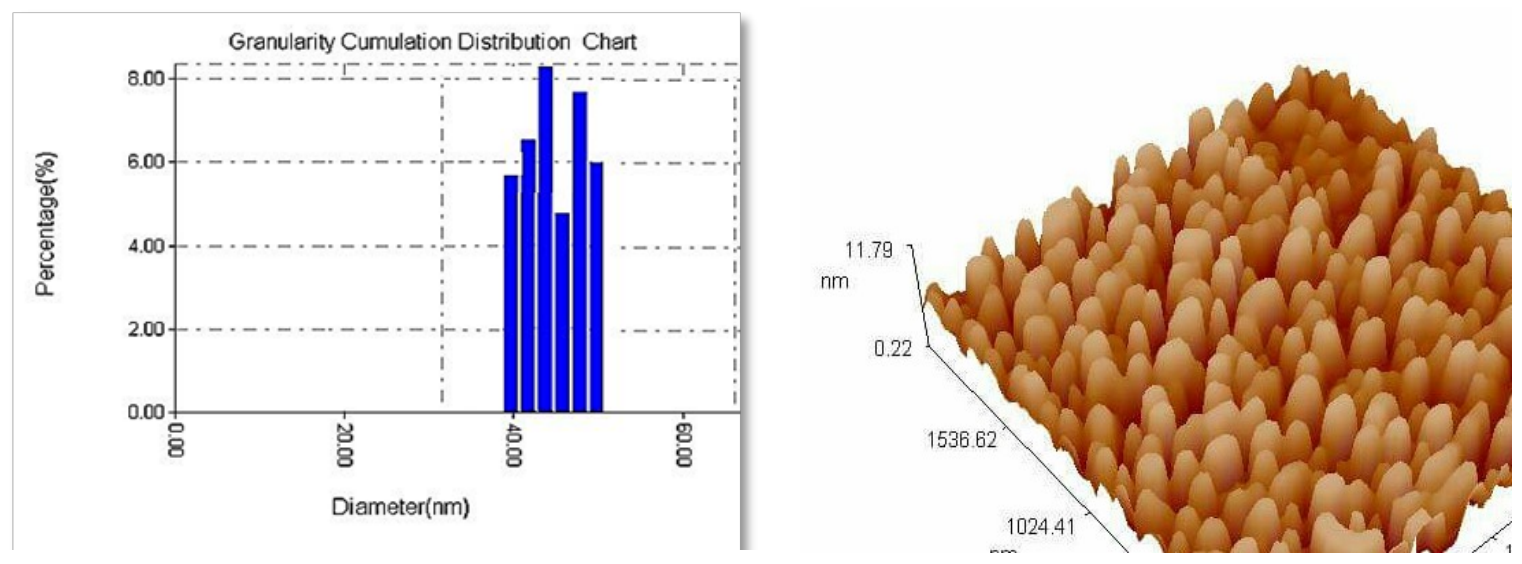

Figure 2. AFM microscope shows the diameter distribution of NiO NPs.

\section{Experimental Part}

Potassium nickel sulfate hexahydrate $\mathrm{K} 2$ (Ni $\left.\left(\mathrm{SO}_{4}\right)_{2} \cdot 6 \mathrm{H}_{2} \mathrm{O}\right)$, HCL, deionized water was used to prepare the solutions, All chemical were used purchased from (BDH) and without any purification. $\mathrm{NiO}$ Nanoparticles have been prepared by photo irradiation method, irradiation cell, as in figure 1 was used to irradiate complexes of nickel as sources of nickel oxide nanoparticles. Immersed UV source $(125 \mathrm{~W}$ mercury medium pressure lamp) is used with maximum light intensity at $365 \mathrm{~nm}$. The cell contains a quartz tube as a jacket for immersion UV source in the complex solution of nickel. Pyrex tube is used as a reactor. The reactor is cooled by ice bath to avoid the temperature rising as a result of the UV irradiation. $0.3 \mathrm{~g}$ of $\mathrm{K}_{2} \quad(\mathrm{Ni}$ $\left.\left(\mathrm{SO}_{4}\right)_{2} \cdot 6 \mathrm{H}_{2} \mathrm{O}\right)$ is dissolved in $30 \mathrm{ml}$ of deionized water and 3 drops of $(37 \%)$ HCL have been add, then, the solution is irradiated by photo cell for $30 \mathrm{~min}$. NiOOH is precipitate as a brown color, it is separated and washed several time with deionize water (all steps done with centrifuge then decantation). The precipitate has dried for one day and calcined at $450^{\circ} \mathrm{C}$ for $2 \mathrm{~h}$. A blackbrown precipitate of nickel oxide nanoparticle is obtained.

\section{Results and discussion}

Characterization of the as-prepared $\mathrm{NiO}$ nanoparticles were carried out by atomic force microscope (AFM), scanning electron microscopy (SEM), transmission electron microscopy (TEM) and X-ray diffraction (XRD) have been used to determine mean particles size, morphology, distribution, crystallinity and purity of nickel oxide NPs.

Mean diameter for NPs of nickel oxide NPs were shown in figure 2 . The result shows the mean diameter of NiO NPs is $(51.91 \mathrm{~nm})$ and the diameter $(40 \mathrm{~nm})$ is the smaller diameter that obtained and the nanoparticle with mean diameter of $(50 \mathrm{~nm})$ consider the mainly diameter. The XRD pattern of the as prepared samples figure 3 shown that the as grown $\mathrm{NiO}$ nanoparticles are in hexagonal phase.

The TEM image figure 4 is a useful approach to estimate the mean size, morphology and distribution of NPs. The result shows the mean particle size of NiO NPs is about $(20 \mathrm{~nm})$. The morphological studies of synthesized NiO NPs were analyzed by scanning electron microscopy SEM as shown in figure 5. It was identified that the shapes of the NiO NPs appeared like hexagonal with rough surfaces. All the nanoparticles have been well separated and without agglomeration was noticed. The strength of magnetic field of samples were determined by using probe joined with gaussmeter it is found that there is around 5-6 G for whale samples.

The nickel oxide NPs synthesized using photo irradiation method was tested for antibacterial activity by agar disc diffusion method against klebsiella and Escherichia coli. After incubation at $37^{\circ} \mathrm{C}$ for 24 hours, the different level of inhibition zone of bacteria was 


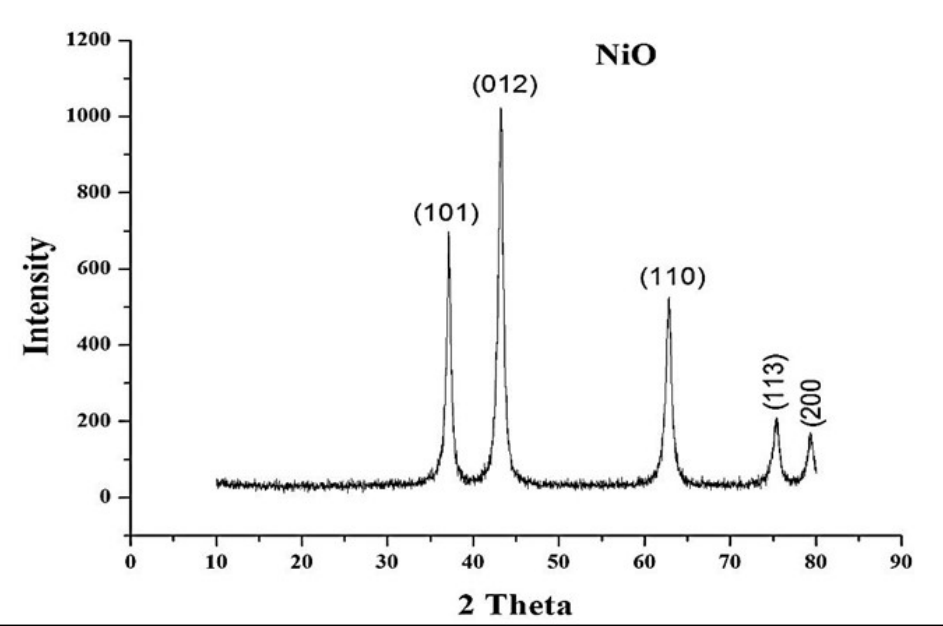

Figure 3. XRD pattern of as synthesized of NiO NPs.
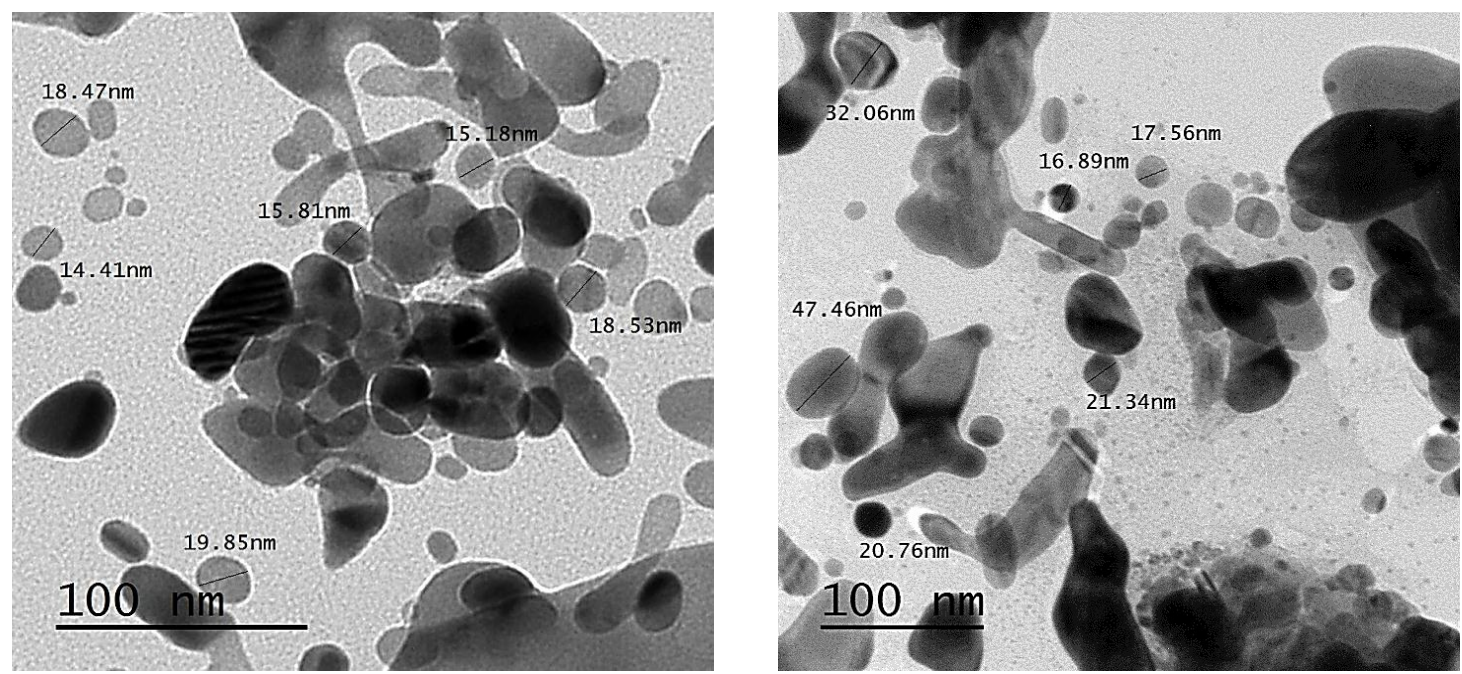

Figure 4. TEM image of Nickel Oxide NiO NPs.
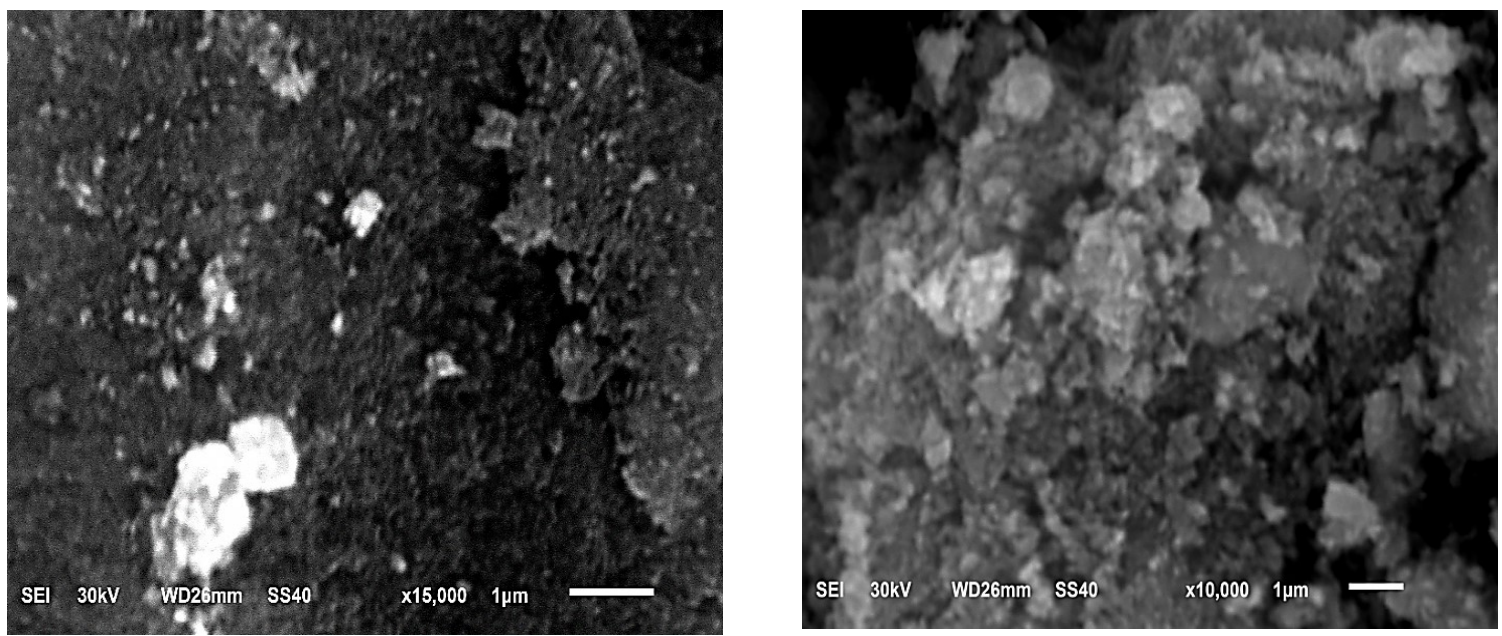

Figure 5. SEM image of $\mathrm{NiO}$.

measured as shown in table 1. Figure 6 shows the inhibition zone of bacterial growth on agar plates by using NiO NPs nanostructure particles of $\mathrm{NiO}$ were successfully synthesized through photo irradiation 

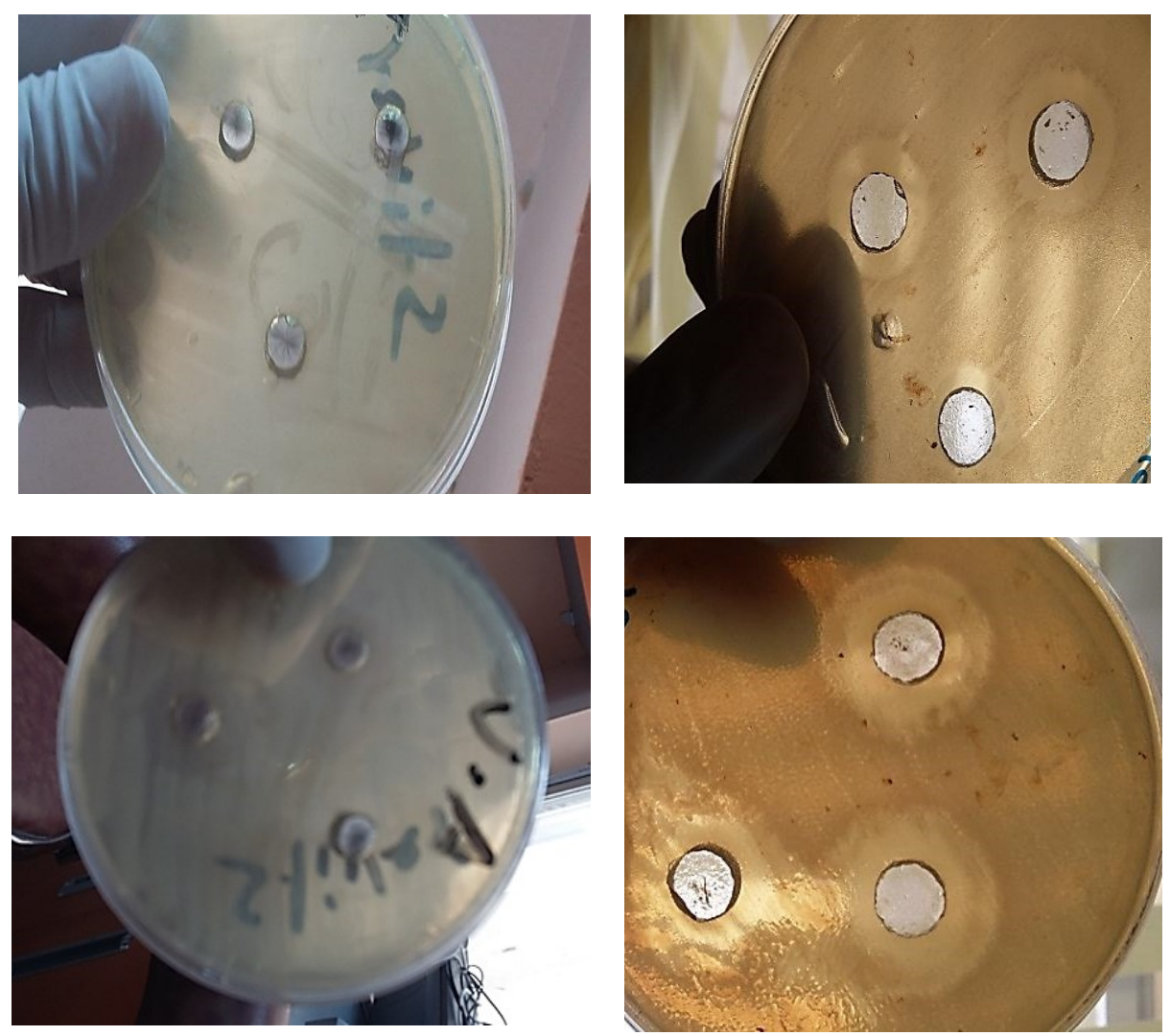

Figure 6. The inhibition zone of bacterial growth on agar plates by using NiO NPs as antibacterial .

Table 1. Inhibition Zone (mm) of synthesis nanoparticles $(\mu \mathrm{l})$.

\begin{tabular}{cc}
\hline Type of Bacteria & Inhibition zone $(\mathrm{mm})$ of NiO NPs \\
\hline klebsiella & 14 \\
Escherichia coli & 16 \\
\hline
\end{tabular}

method. XRD results estimate the average particle size of NiO NPs was (33.92 nm). It also confirms the high degree crystallinity nature and purity of the prepared sample. TEM confirms that the particles for $\mathrm{NiO}$ was confirmed at nanoscale. The morphological studies and appearance of synthesized $\mathrm{NiO}$ nanoparticles and analyzed by SEM are showed that some particles are in spherical and some are in semi spherical shapes. From the results, this simple have a novelty and cost effective and will be useful for industries for the preparation of $\mathrm{NiO}$ nano-sized particles.

Table 1 indicates that the nanoparticles of $\mathrm{NiO}$ were showed inhibiting effect towards different types of bacteria. The activity of nanoparticles against bacteria has been reported by several studies, but the mechanism of the bactericidal effect of nanoparticles is not understood completely. But studies suggest that when bacteria treated with NiO NPs. The nanoparticle attach to surface membrane ,then many change took place in its membrane morphology and disturb its power function such as permeability, produced a significant increase in its permeability affecting proper transport through the plasma membrane, resulting cell death. It have believed that the penetration of nanoparticle inside the bacteria casing damage by interaction with sulfur and phosphorous containing compounds such as DNA. The replication ability have lost and cellular proteins become an inactive after nanoparticle treatment. Another reason would be the release of $\mathrm{Ni}$ ions from nanoparticles, the electrostatic attraction between the positive charge of NPs and negative charge of the cell membrane of microorganism. It is also known that NiO NPs may have penetrated the strain's cell wall and altered the cell membrane and internal cellular components leading to death of the cell. Similarly, few studies suggested that metal oxide inhibits microbial pathogens ' growth completely, In this present study it was shown that nickel oxide nanoparticles gave a very high efficacy against the type of bacteria as shown in Table 1 and Figure 6. This result is Consistent and superior on many previous studies [23-27].

\section{Conclusions}

NiO NPs was synthesized via photolysis method using simple materials. This method is an easy and low cost for synthesis nanoparticles. XRD was used to characterize the crystal structure of nickel oxide nanoparticles. Average particles size was in rang (20-50) that is calculated from SEM, AFM and TEM. NiO NPs have strong activity against bacteria and this activity is influenced by the size of nanoparticles, because of it can 
easily reach the nuclear content of bacteria and notable surface area. The outcome of magnetization measurement, display a weak magnetic behavior. Thus the contact with bacteria were the highest and it can be reason behind the strong activity against bacteria and then more small particles make large inhibition zones.

\section{References}

1. J W Costerton, Annu. Rev. Microbiol. 41, 1 (1987) 435.

2. H Pang, Bacterial biofilms Chem Comm. 48 (2009) 7542-754.

3. M Oussalah, Food control. 18, 5 (2007) 414.

4. P S Mead, Emerg Infect Dis. 5, 5 (1999) 607.

5. G A Burton, G Douglas, and R L Guy, Appl. Environ. Microbiol. 53, 4 (1987) 633.

6. A R Shahverdi, Biology and Medicine 3, 2 (2007) 168.

7. A Jokar and A Ramazani, Iran. J. Phys. Res. 18, 3 (2018) 401.

8. N Dharmaraj, Mater. Chem. phys. 87, 1 (2004) 5.

9. Y Changzhou, J. Mater. Chem. 19, 32 (2009) 5772.

10. W R Jarvis and J M William, Antimicrob. Chemother. Suppl.A 29 (1992) 19.

11. J W Chow, Annals of internal medicine. 115, 8 (1991) 585.

12. K K Lai,Medicine. 80, 2 (2001) 113.

13. A R Hoffmaster, Proc Natl AcadSci. 101, 22 (2004) 8449.

14. A M Rheima, et al., Journal of Southwest Jiaotong University 54, 5 (2019) 34.

15. D H Hussain, et al., Egyptian Journal of Chemistry

\section{Acknowledgments}

A special thanks to the University of Wasit, Al-Karkh University of Science, Al-Qasim green University and University of Kerbala for supporting this work..

62, 11 (2019) 417.

16. X Song and G Lian, J. Phys. Chem. C. 112, 39 (2008) 15299.

17. S V Ganachari, Recent Res. Sci. Technol. 4, 4 (2012) 16.

18. B M Lund, Lancet. 336. 20 (1990) 982.

19. D A Wruck and M J Rubin, Electrochem. Soc. 140, 4 (1993) 1097.

20. S J Yamada, Appl. Phys. 63, 6 (1988) 2116.

21. K Yoshimura, M Takeshi, and T Sakae. Jpn. J. Appl. Phys. 34 (1995) 2440.

22. X Wang, Nanotechnology 6, 1 (2004) 37.

23. S D Sarker, L Nahar, and Y Kumarasamy. Methods 42 (2007) 321.

24. N Naz and M Z Iqbal. Sci. Int. (Lahore) 23 (2011) 27.

25. J Nathanael, J H Lee, D Mangalaraj, S I Hong, and Y H Rhee, Powder Technol 228 (2012) 410.

26. K Soo-Hwan, H S Lee, D S Ryu, S J Choi, D S Lee, J Microbial Biotechnol 39 (2011) 77.

27. Ali H. A. Jalaukan et. al., Iranian Journal of Materials Science \& Engineering 16, 4 (2019) 53. 